\title{
Making Dentition Model from Negative Dental Impression in Implant Treatment
}

\author{
Fabricación de un Modelo de Dentición a Partir de una \\ Impresión Dental Negativa para el Tratamiento con Implantes
}

Koojoo Kwon*; Min-Su Kim* \& Byeong-Soek Shin*

KWON, K.; KIM, M. S. \& SHIN, B. S. Making dentition model from negative dental impression in implant treatment. Int. J. Morphol., 34(4):1404-1410, 2016.

SUMMARY: In dental implant treatment or corrective surgery, a dental plaster model is produced to recognize the shape of the teeth. Understanding this dentition model is an important issue in prosthetic dentistry and craniomaxillofacial surgery. However, dental models are time consuming and costly to produce via traditional casting methods. We provide a method which makes dentition model from both a positive and a negative dental impression. Also we produce a system for making a digital dentition model using computed tomography (CT) data during the impression process. Additionally, the system allows the digital dentition model to be realized via a three-dimensional printer. The result simplifies production of the dentition model, since a physical model can be produced directly from an impression of the patient. Our system can simplify the dentition process and treatment intervention involved in making a dentition model. The digital model enables clinicians to manage the patient's cumulative data and to predict changes during the course of treatment.

KEY WORDS: Dental impression; Dentition model; Implant treatment; Prosthetic dentistry; Three-dimensional printing;

\section{INTRODUCTION}

Many dentition models have been used in dental treatment. Orthodontic surgery corrects congenital or acquired deformations of the skull and face, including maxillofacial deformities, congenital malformations and deformations of the jaw joint. Implant treatment is important in orthodontic surgery, since it enables the replacement of natural teeth (Xia et al., 2000; Xia et al., 2005; Zachow et al., 2006; Swennen et al., 2007). Although the success of technical aspects is important in dental surgery, effective management of the treatment schedule is also essential, since a dentist must consider the intermediate results in determining treatment prognosis. Significant improvements in dental fixing technology and impression materials provide benefits in establishing a preoperative plan; however, the treatment process remains almost the same as in previous methods (Gateno et al., 2003; Swennen et al., 2009; Xia et al., 2001, Bell \& Guerrero, 2007). Existing dental diagnostic methods for establishing treatment plans involve a dental cast, and obtaining photographic or X-ray images from the front and side of the patient's face (Bookstein et al., 1991; Proffit \& Ackerman, 1992). Establishing the relationship between the dentition in three-dimensional (3D) space by using the two-dimensional (2D) image obtained from the patient is very important during the treatment process (Lin \& White, 1995; Beyer \& Lindauer, 1998). Some computer-based surgical simulation software displays the 3D facial model for easy identification of complex anatomical structures (Gateno et al., 2003; Swennen et al., 2009; Xia et al., 2001; Gateno et al., 2007). Recently, computed tomography (CT) and magnetic resonance imaging (MRI) have been used in dentistry to recognize the 3D relationships between face and teeth (Altobelli et al., 1993; Santler et al., 1998; Gaggl et al., 1999; Ploder et al., 2002; Abbaszadeh et al., 2000).

Gateno et al. (2003), proposed a computerized composite skull model that combines a 3D CT bone model with digital dental models of the skull. A high-resolution digital dental model was produced from geometric data obtained using a 3D laser surface-scanner. However, the method is not suitable for detailed verification of the connectivity among the teeth, since the scanned information can be obtained only for the outside. Swennen et al. (2009), proposed a process for producing an

\footnotetext{
* Department of Computer Science and Information Engineering, Inha University, Incheon, Korea.
} 
augmented virtual skull model using volume data, which is reconstructed from stacking $2 \mathrm{D}$ images, obtained from a high-resolution cone-beam computed tomography (CBCT). This digital skull model can provide accurate shape of dentition. However, the dentition models still require a hand-polishing step because it is difficult to distinguish the teeth from bone areas. Furthermore, it is difficult to obtain an accurate model if diffusion occurs with the metal material inserted into the tooth. In general, existing approaches for making a digital dentition model are complex and therefore time consuming.

We propose a digital dentition model reconstruction method and STL (STereoLithography) file conversion system for 3D printing of the reconstructed model. Firstly, we create volume data by stacking images that have been scanned directly from the patient using CBCT. Then, we reconstruct the surface geometry from the volume data. If the volume data include a negative dental impression model, we makes a positive dentition model from this negative dental impression. Lastly, these geometry converts to STL format. Also we produce a dentition model by printing this STL file in three dimensions. Our system includes both the CBCT scanning device and a 3D printer, and software for converting the volume data to the STL file. Also the cost of our system is relatively lower than the previous dental system. We can apply the dentition model directly in the treatment process, since there is no manual procedure for modifying it with materials such as gypsum. The proposed system replaces a traditional plaster model with a digital representation of the teeth, thereby shortening the time required to produce a dental impression model. It also facilitates convenient and rapid management of the treatment process, since the digital dentition model enables us to utilize morphological analysis. Our system helps dentist to communicate with the patient interactively and ensures that treatment proceeds quickly and smoothly.

In Section 2, we describe the surface model reconstruction method using $\mathrm{CBCT}$ data and explain the STL file export system for producing the dental model. The results are shown in Section 3. Lastly, we discuss and conclude our work.

\section{MATERIAL AND METHOD}

Material and process. We use CBCT images scanned from a dental maxillary (or mandibular) plaster model or a dental impression. These images are stacked transversely direction to represent the volume and geometry of the oral cavity in three dimensions. Figure 1 shows the procedure for producing our dentition model. Volume data are acquired from the CBCT device as shown in the Figure 1. Our system contains two-layered separation table for scanning both maxillary and mandibular dentition impressions. Volume data are converted into STL format as shown in the middle of Figure 1, and a 3D printer constructs a dentition model from the STL file, as shown in the right of Figure 1.

In order to create a surface model of the object represented by the volume data, the density values of the object have to be distinguished from those of background material. The density values of the volume data depend on the scanning environment. We therefore use several materials having different density values in order to distinguish the dental impression from the separation table. We set the plastic separation table, as shown in the left of Figure 1, to differentiate between maxillary and mandibular dental impressions, and a sponge is inserted between the impression and the table, since a sponge has low density. Our system can use both dental maxillary (or mandibular) plaster models and dental impression as input objects. The dental impression is produced using a container designed to fit directly over the patient's dental arches. This plaster model is produced by filling the impression tray with dental molding materials such as plaster.

Making surface geometry and converting to STL format. In order to print a 3D dentition model, the surface geometry of an object should be reconstructed. Since the volume data do not contain geometric information, a surface extraction process is necessary to convert the volume into the surface geometry. This process consists of three sub-steps: region selection, surface extraction and STL format conversion (see the middle of Fig. 1).

The volume data consist of: dental impression, separation table and empty space. Empty space has very low density, and the dental impression and the table have their own density ranges. We can therefore define the dentition model by using a histogram-based binary thresholding method (Glasbey et al., 1993), which is widely used in image processing.

Figure 2 shows the process to find an object within the volume data by using the histogram. The four histograms are derived from several dentition volumes (see the top of Fig. 2). The graph shows the approximate shape of these histograms drew (see the bottom right of Fig. 2). All cases show left $(\mathrm{L})$ and right $(\mathrm{R})$ peaks separated by a valley area. The density value th $\mathrm{m}_{\mathrm{m}}$ of the object to be identified is located in the valley region of the histogram curve. This value can be used as a threshold for distinguishing two objects, and is therefore the density of the target object. 


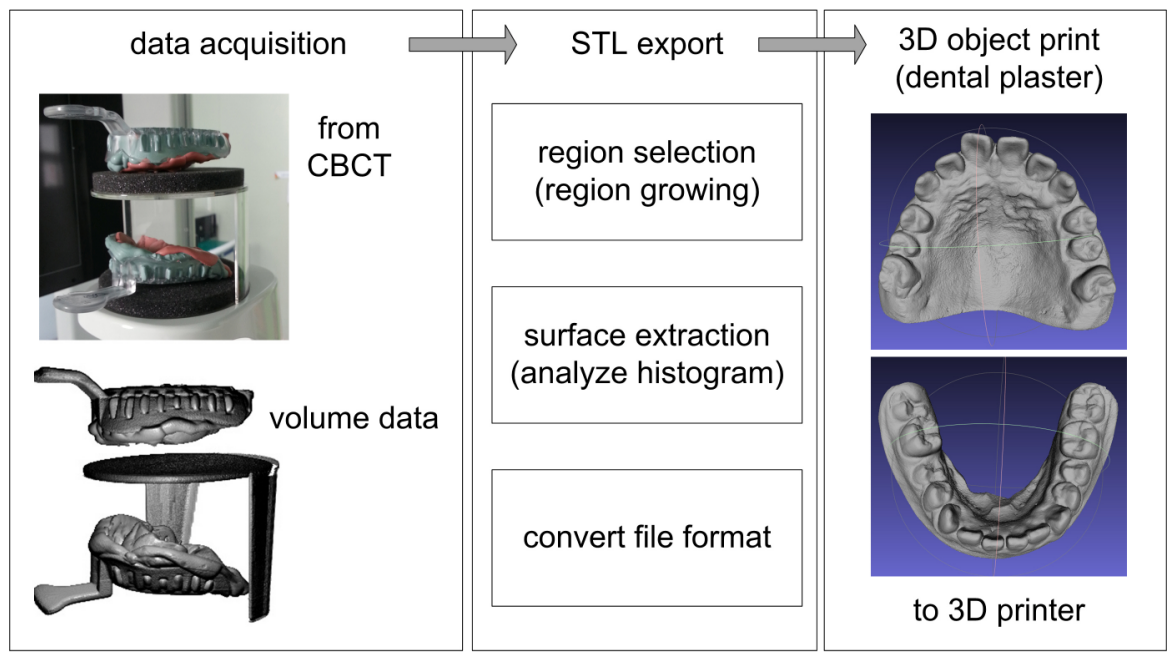

Fig. 1. Process of producing a dentition model. Data acquisition (left), STL conversion (middle) and dental plaster printing (right)
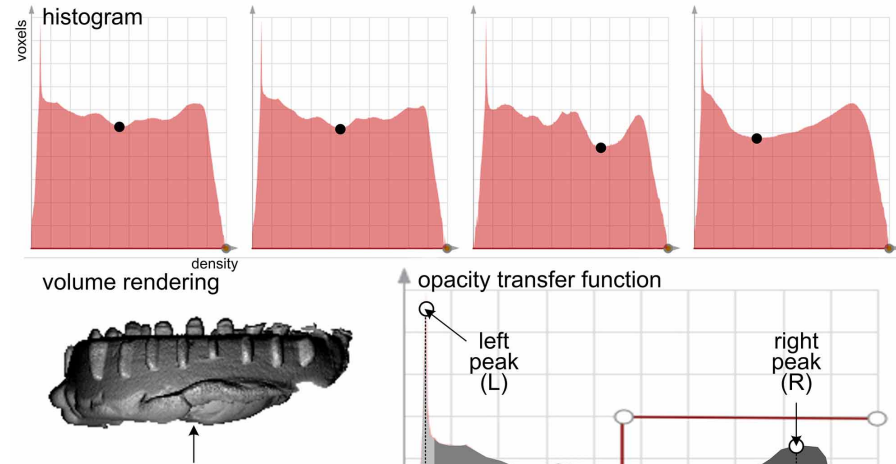

volume rendering result using OTF(value $\mathrm{m}$ )
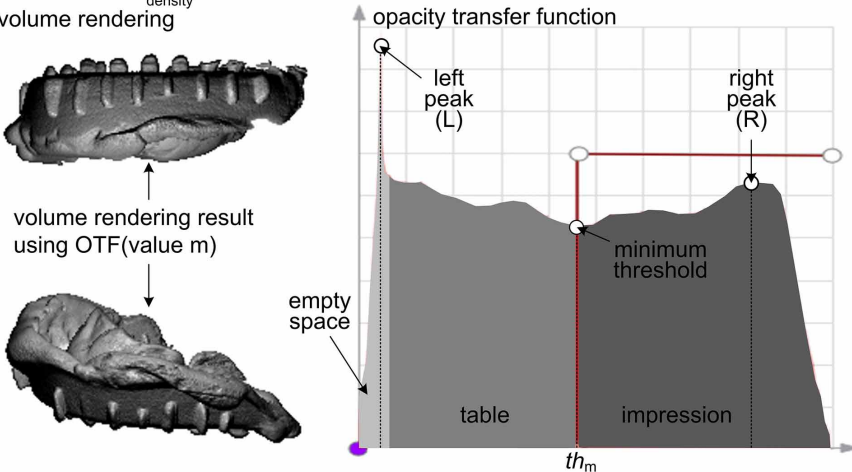

Fig. 2. Histogram of dentition model (top), volume rendering result (bottom left) and opacity transfer function (bottom right). The data show two peaks, left and right, which represent density values for each object. The minimum value (th ) can be used as a threshold for distinguishing two objects
Assuming that the histogram curve is the $n$-order function, we can solve this function by the second derivative equation in order to find the inflection point of the curve. Among several inflection points that have concave down direction, the lowest $y$ is the value $t_{\mathrm{m}}$. After finding the object corresponding to the value $\mathrm{th}_{\mathrm{m}}$, a surface model of this object is created using the marching cubes (MC) algorithm (Lorensen \& Cline, 1987).

If the histogram curve is not determined due to irregularly distributed densities, as shown in the left of Figure 3, the threshold $\left(\mathrm{th}_{\mathrm{m}}\right)$ cannot be identified. In our system, we simplify the curve to a smaller number of resampling points by applying a large sampling interval to the $x$-axis.
The STL format is commonly used to represent surface models in 3D geometry-based systems such as computer-aided design (CAD) or a 3D printer. The surface of an object is divided into a set of tessellated triangles, and the face of each triangle is determined by a normal vector with three vertices. Therefore, the STL file includes a set of coordinates for each vertex and normal vectors of triangles. The STL file can be saved in BYTE or BINARY formats. The BYTE format has good readability, since it is represented by characters. However, the resulting file requires a large amount of memory space and takes longer to generate and also to transfer for $3 \mathrm{D}$ printing. The proposed system uses the BINARY format, since the smaller file size saves on memory space and reduces transmission time. 
Positive plaster model and negative dental impression. A positive dental plaster model and a negative dental impression are frequently used in the dental impression process. The negative dental impression is obtained immediately using CBCT, after scanning the patient's head. The positive dental plaster model can be used as input data after filling the mold produced by the dental impression.

Firstly, we have to separate the upper and lower regions of the volume data. The center position of the zaxis is used to differentiate maxillary and mandibular regions, since the maxillary model on the separation table is located on the center position of the volume height.

In the case of a negative dental impression, the impression model has to be converted into STL files after filling the inside of the negative imprint. To do this, we use the seed point-based region growing (SRG) algorithm (Adams \& Bischof, 1994). A seed point is determined within the negative imprint, which then propagates in the direction of its six neighbors until reaching the boundary.

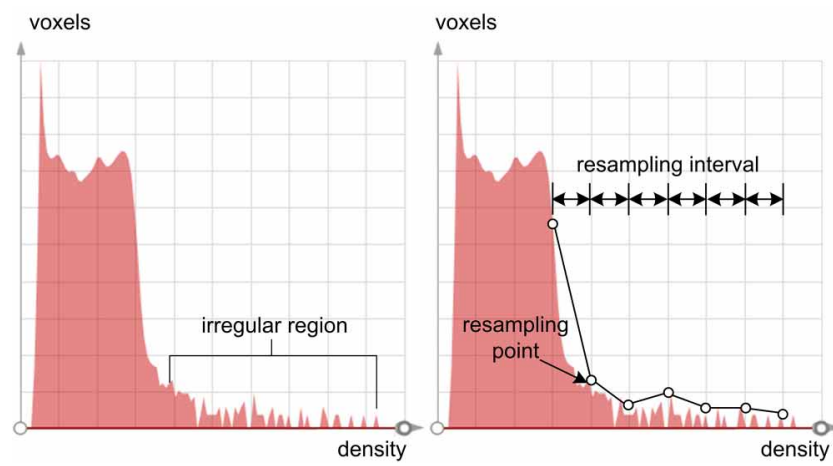

Fig. 3. Irregularly distributed region of the histogram (left) and reduced resampling points of the histogram curve using large interval (right)

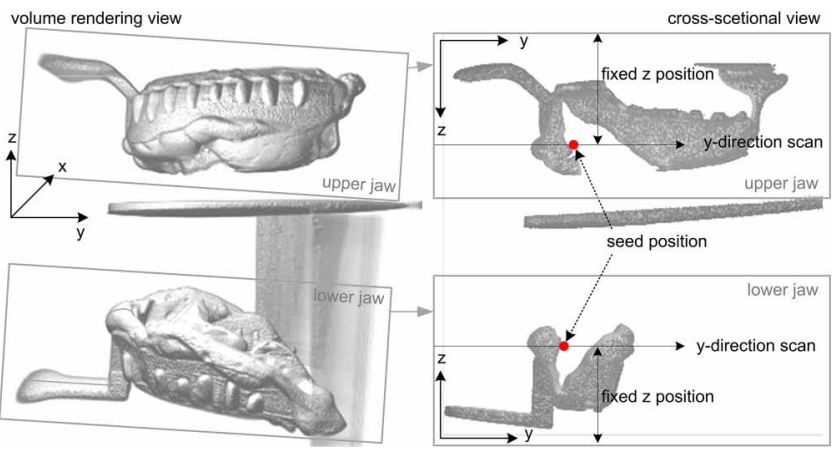

Fig. 4. Identification of seed point in a dental impression. Volume rendering image (left) and the cross-sectional image at the center of the $x$-axis (right)
Figure 4 shows the process for determining the seed point inside the imprint. All objects used in our system can easily establish a seed point located inside the imprint, since the dental impression is located at the same place on the separation table in our scanning system (see Fig. 1). The position of the seed point is calculated by $z, x$ and $y$ axes in order. We perform the following steps:

1. Set a distance larger than the thickness of the dentition imprint from the table as $z$ (tooth position). We set the $z$ distance less than the height of the tooth on the table, since the SRG algorithm does not function if the seed point is buried inside the dental impression.

2. Set the center position of the $\mathrm{x}$-axis is the $x$ value (the position of foretooth)

3. Scan the y direction based on fixed $x$ and $z$ positions established in (1) and (2). We select a y position in the first empty space beyond the object area, since voxels are always arranged in the order of direction y as: empty area - object area - empty area.

The region is propagated from the seed point to the boundary of the volume. The SRG algorithm proceeds similarly to water filling a bowl, since the shape of the dentition imprint is an open structure without a ceiling. Therefore, we only have to limit the $z$-axis in order to prevent overflow.

\section{RESULTS}

The environment of our system is an Intel Core ${ }^{\mathrm{TM}}$ i7-2600 3.4GHz CPU with 8 GB of RAM. The system has a 64-bit operating system and the algorithm is tested with 64-bit applications. The volume of the dental dataset is $600 \times 600 \times 600$ and the voxel size is $0.2 \mathrm{~mm}$. The performance of each step is shown in Table I.

As shown in Table I, the total time to calculate the surface geometry after acquiring the volume data is approximately 20 seconds even using BYTE format. This implies that our system is much faster than the other methods while producing a dental impression manually and thereby enabling rapid verification of the patient's dentition status.

Figure 5 shows the result of making the STL from dental volume data, allowing verification of the shape of teeth, connectivity of dentition and the palatine folds. 
Table I. Performance and capacity at each step using 64-bit system

\begin{tabular}{ccccc}
\hline Type & Total time & Initialize & Export STL (include MC) & Capacity \\
\hline BINARY & $17.96 \mathrm{sec}$. & $0.42 \mathrm{sec}$. & $17.54 \mathrm{sec}$. & $21.07 \mathrm{MB}$ \\
BYTE & $21.55 \mathrm{sec}$. & $0.48 \mathrm{sec}$. & $21.07 \mathrm{sec}$. & $86.6 \mathrm{MB}$ \\
\hline
\end{tabular}

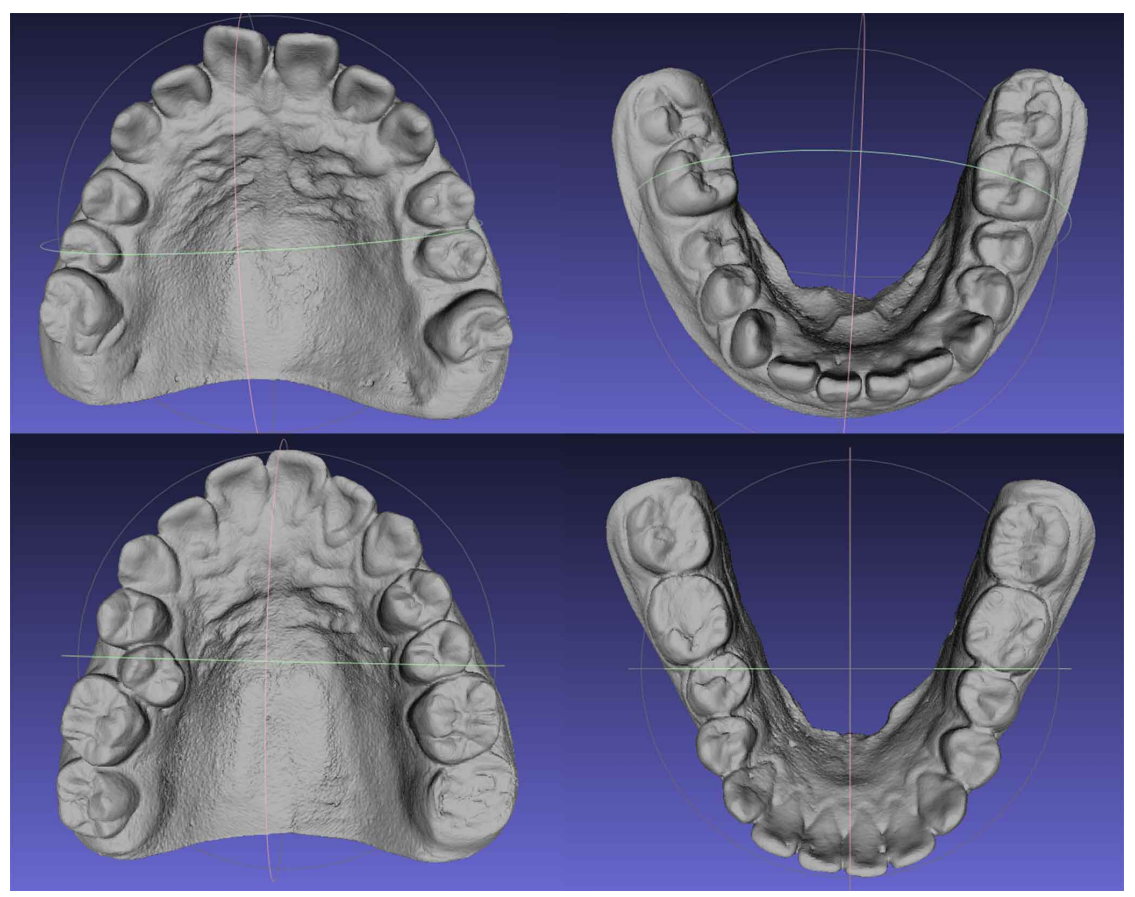

Fig. 5. Result of extracting STL. A surface model from volume data of maxillary plaster model (left column) and mandibular plaster model (right column)

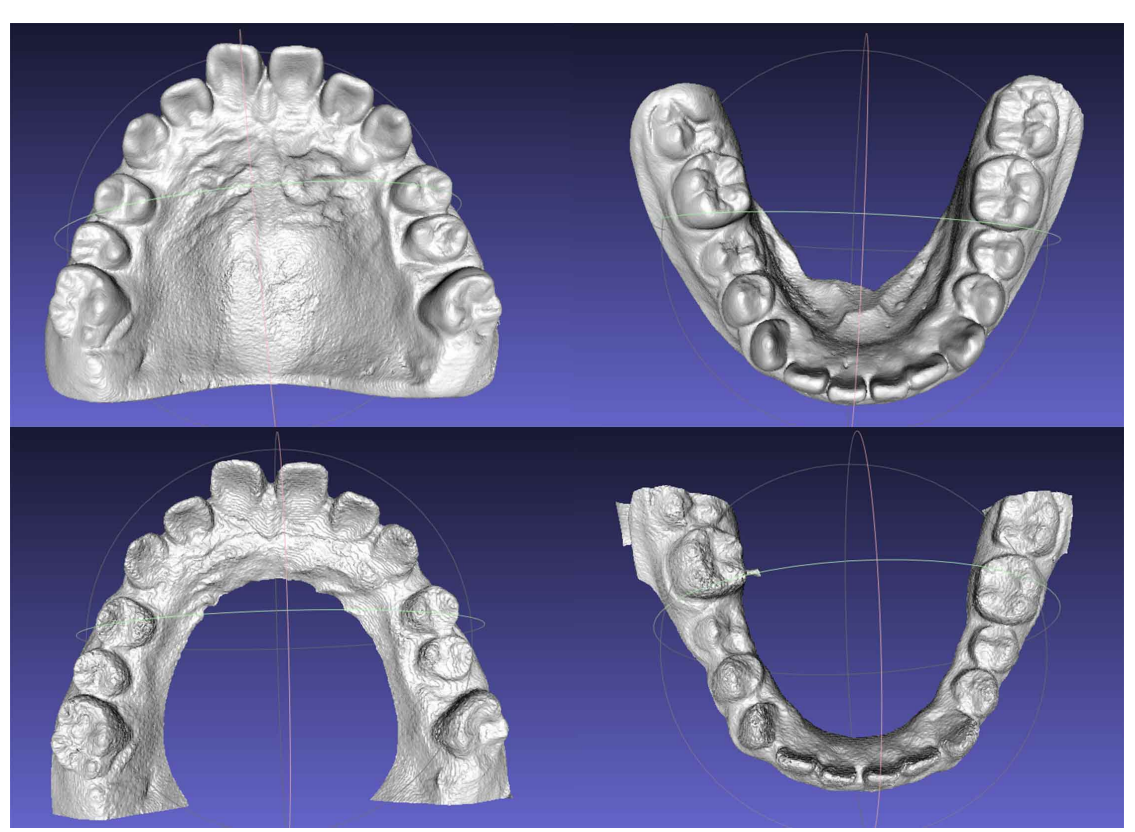

Fig. 6. Surface model of maxillary and mandibular plaster model (top) and that obtained by making of maxillary and mandibular data from negative dental impression (bottom).

\section{DISCUSSION}

The top row of Figure 6 shows the surface of positive maxillary and mandibular plaster models, and the bottom row shows the surface obtained by making maxillary and mandibular data from a negative dental impression. The left and right columns are derived from the maxillary and mandibular model respectively. The STL models in the bottom row are extracted by using the SRG algorithm. The shape of dentition is the same for the top and bottom rows.

Figure 7 compares the shape of the negative dental impression obtained from a patient with the digital surface model rendered via our system. This dental impression is acquired from a mandibular dentition of a patient; the shape of dentition appears uncertain, since it is a negative model. However, it can be seen that the mandibular dentition is clearly represented by the surface model (the Fig. 7 B).

The seed point is established inside the negative dental impression and expands outward in six directions to connected regions. If there are no walls to limit expansion from the seed area, the dentition model will flow over the negative imprint. In the present model, five walls (front, back, right, left and top) are established around the negative dental impression.

A dental plaster model obtained directly from the patient is commonly used in order to understand the shape of the teeth during implant treatment or facial prosthetic surgery. However, dental plaster models are time consuming and costly to produce manually. We propose a method which converts from a negative dental impression to a positive dental plaster model. Also we provide a system for making a digital dentition model using 


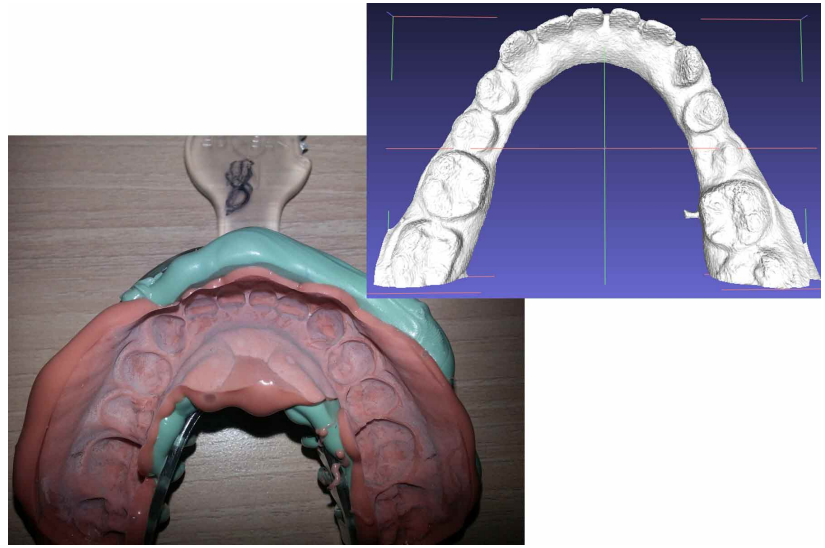

Fig. 7. Negative dental impression from a patient (left) and corresponding digital surface model (right).

CBCT data acquired directly from the patient, which can be realized via a 3D printer. Our system can simplify the process and treatment intervention involved in making a dentition model. Furthermore, the use of a digital model enables management and analysis of the patient's data series and prediction of changes in dentition.

\section{ACKNOWLEDGEMENTS}

This work was supported by the National Research Foundation of Korea (NRF) grant funded by the Korea government (MSIP) (No. 2015R1A2A2A01008248). This research was supported by Next-Generation Information Computing Development Program through the National Research Foundation of Korea (NRF) funded by the Ministry of Science, ICT \& Future Planning (2012M3C4A7032781). This research was supported by Basic Science Research Program through the National Research Foundation of Korea (NRF) fundedby the Ministry of Education (2015R1D1A4A01020277). The dataset for experiment is provided from Ewoosoft Co., Ltd.

KWON, K.; KIM, M. S. \& SHIN, B. S. Fabricación de un modelo de dentición a partir de una impresión dental negativa para el tratamiento con implantes. Int. J. Morphol., 34(4):1404-1410, 2016.

RESUMEN: En el tratamiento de implantes dentales, o cirugía correctiva, se produce un modelo de yeso dental para reconocer la forma de los dientes. La comprensión de este modelo de dentición es un tema importante en la odontología protésica y la cirugía craniomaxilofacial. Sin embargo, los modelos dentales con- sumen tiempo y son costosos de producir a través de los métodos tradicionales de fundición. Proporcionamos un método que hace que el modelo de dentición sea tanto positivo como negativo. También proponemos un sistema para hacer un modelo de dentición digital utilizando datos de tomografía computarizada (TC) durante el proceso de impresión. Además, el sistema permite que el modelo de dentición digital se imprima a través de una impresora tridimensional. El resultado simplifica la producción del modelo de dentición, ya que un modelo físico puede ser producido directamente a partir de una impresión del paciente. Nuestro sistema puede simplificar el proceso de dentición y la intervención de tratamiento involucrados en la elaboración de un modelo de dentición. El modelo digital permite a los médicos manejar los datos acumulados del paciente y predecir los cambios durante el curso del tratamiento.

PALABRAS CLAVE: Impresión dental; Modelo de la dentición; Tratamiento del implante; Odontología protésica; Impresión tridimensional.

\section{REFERENCES}

Adams, R. \& Bischof, L. Seeded region growing. I. E. E. E. Trans. Pattern Anal. Mach. Intell., 16(6):641-7, 1994.

Abbaszadeh, K.; Heffez, L. B. \& Mafee, M. F. Effect of interference of metallic objects on interpretation of T1-weighted magnetic resonance images in the maxillofacial region. Oral Surg. Oral Med. Oral Pathol. Oral Radiol. Endod., 89(6):759-65, 2000.

Altobelli, D. E.; Kikinis, R.; Mulliken, J. B.; Cline, H.; Lorensen, W. \& Jolesz, F. Computer-assisted three-dimensional planning in craniofacial surgery. Plast. Reconstr. Surg., 92(4):576-85, 1993.

Bell, W. H. \& Guerrero, C. A. Distraction Osteogenesis of the Facial Skeleton. Hamilton, BC Decker, 2007.

Bookstein, F. L.; Grayson, B.; Cutting, C. B.; Kim, H. C. \& McCarthy, J. G. Landmarks in three dimensions: reconstruction from cephalograms versus direct observation. Am. J. Orthod. Dentofacial Orthop., 100(2):133-40, 1991.

Beyer, J. W. \& Lindauer, S. J. Evaluation of dental midline position. Semin. Orthod., 4(3):146-52, 1998.

Gaggl, A.; Schultes, G.; Santler, G.; Kärcher, H. \& Simbrunner, J. Clinical and magnetic resonance findings in the temporomandibular joints of patients before and after orthognathic surgery. Br. J. Oral Maxillofac. Surg., 37(1):41$5,1999$.

Gateno, J.; Xia, J.; Teichgraeber, J. F. \& Rosen, A. A new technique for the creation of a computerized composite skull model. J. Oral Maxillofac. Surg., 61(2):222-7, 2003. 
Gateno, J.; Xia, J. J.; Teichgraeber, J. F.; Christensen, A. M.; Lemoine, J. J.; Liebschner, M. A.; Gliddon, M. J. \& Briggs, M. E. Clinical feasibility of computer-aided surgical simulation (CASS) in the treatment of complex craniomaxillofacial deformities. J. Oral Maxillofac. Surg., 65(4):728-34, 2007.

Glasbey, C. A. An analysis of histogram-based thresholding algorithms. CVGIP: Gr. Models Image Process., 55(6):532-7, 1993.

Lin, S. Y., \& White, G. E. Mandibular position and head posture as a function of eye dominance. J. Clin. Pediatr. Dent., 20(2):13340, 1995 .

Lorensen, W. E. \& Cline, H. E. Marching cubes: A high resolution 3D surface construction algorithm. ACM SIGGRAPH Comput. Gr., 21(4):163-9, 1987.

Ploder, O.; Klug, C.; Backfrieder, W.; Voracek, M.; Czerny, C. \& Tschabitscher, M. 2D- and 3D-based measurements of orbital floor fractures from CT scans. J. Craniomaxillofac. Surg., 30(3):153-9, 2002.

Proffit, W. R. \& Ackerman, J. L. Contemporary Orthodontics. $2^{\text {nd }}$ ed. St. Louis, Mosby-Year Book, 1992. pp.139-85.

Santler, G.; Kärcher, H. \& Ruda, C. Indications and limitations of three-dimensional models in cranio-maxillofacial surgery. $J$. Craniomaxillofac. Surg., 26(1):11-6, 1998.

Swennen, G. R.; Barth, E. L.; Eulzer, C. \& Schutyser, F. The use of a new 3D splint and double CT scan procedure to obtain an accurate anatomic virtual augmented model of the skull. Int. J. Oral Maxillofac. Surg., 36(2):146-52, 2007.

Swennen, G. R.; Mollemans, W.; De Clercq, C.; Abeloos, J.; Lamoral, P.; Lippens, F.; Neyt, N.; Casselman, J. \& Schutyser, F. A cone-beam computed tomography triple scan procedure to obtain a three-dimensional augmented virtual skull model appropriate for orthognathic surgery planning. J. Craniofac. Surg., 20(2):297-307, 2009.

Xia, J.; Ip, H. H.; Samman, N.; Wang, D.; Kot, C. S.; Yeung, R. W. \& Tideman, H. Computer-assisted three-dimensional surgical planning and simulation: 3D virtual osteotomy. Int. J. Oral Maxillofac. Surg., 29(1):11-7, 2000.

Xia, J.; Ip, H. H.; Samman, N.; Wong, H. T.; Gateno, J.; Wang, D.; Yeung, R. W.; Kot, C. S. \& Tideman, H. Three-dimensional virtual-reality surgical planning and soft-tissue prediction for orthognathic surgery. I. E. E. E. Trans. Inf. Technol. Biomed., 5(2):97-107, 2001.

Xia, J. J.; Gateno, J. \& Teichgraeber, J. F. Three-dimensional computer-aided surgical simulation for maxillofacial surgery. Atlas Oral Maxillofac. Surg. Clin. North Am., 13(1):25-39, 2005.
Zachow, S.; Hege, H. C. \& Deuflhard, P. Computer-assisted planning in cranio-maxillofacial surgery. J. Comput. Inf. Technol., 14(1):53-64, 2006.

Correspondence to:

Byeong-Seok Shin

(402-751)

Department of Computer Science and Information Engineering

Inha University

100 Inha-ro, Nam-gu

Incheon

KOREA

Email: bsshin@inha.ac.kr

Received: 11-03-2016

Accepted: 21-10-2016 